ESAIM: PROCEEDINGS, December 2013, Vol. 41, p. 77-94

L. Chupin, A. Münch, Editors

\title{
MATHEMATICAL MODEL OF CANCER GROWTH CONTROLED BY METRONOMIC CHEMOTHERAPIES *
}

\author{
N. André ${ }^{1,2}$, D. Barbolosi ${ }^{1}$, F. Billy ${ }^{3}$, G. Chapuisat $^{4}$, F. Hubert $^{2}$, E. Grenier $^{5}$ \\ AND A. Rovini ${ }^{1}$
}

\begin{abstract}
We propose in this article to compare the efficiency of two chemotherapeutic schedules: the traditional and the metronomic. For this, we develop a new mathematical model describing the growth dynamics of tumor and endothelial cells as well as the impact of molecules as oxygen or vascular endothelial growth factor on this dynamics. The model construction: biological assumptions, description of the equations and their discretization, constitutes the core of the article. Numerical experiments illustrate the expected behavior of the disease under the two chemotherapeutic schedules.

Résumé. Nous proposons dans cet article de comparer l'action de deux protocoles d'administration de chimiothérapie. Pour cela, nous développons un nouveau modèle mathématique décrivant la dynamique de croissance de diverses cellules tumorales et endothéliales et l'impact sur cette dynamique de molécules comme l'oxygène et de facteurs de croissances endothéliales. La construction du modèle, à savoir les hypothèses biologiques sous-jacentes, la description des équations et leur discrétisation constitue le coeur de cet article. Nous donnons quelques illustrations numériques de l'évolution de la maladie sous l'action des deux protocoles thérapeutiques et retrouvons les résultats attendus par les oncologues.
\end{abstract}

\section{INTRODUCTION}

\section{Metronomic chemotherapy.}

Cancer is a major cause of death worldwide and tends to become the first cause of death in the industrialized countries. Many studies were and are still designed to improve the efficacy of existing anti-cancer treatment by optimizing the administration schedule. Based on the concept that the more anti-cancer drug are administered to the patient, the more tumor cells may die, the chemotherapeutic schedule traditionally used in clinical practice consists in the administration of the maximal dose of anti-cancer drugs that can be tolerated by the patient, also called the maximal tolerated dose (MTD). As chemotherapy kills not only cancer cells but any cell in state of rapid proliferation, MTD induces serious toxicities, depending on the type of anti-cancer agent used, and

* This study was supported by Cancéropole PACA.

1 CRO2 - INSERM UMRs 911, Aix-Marseille Université, Faculté de Médecine et Faculté de Pharmacie, 27 Boulevard Jean MOULIN - 13385 Marseille cedex 05, FRANCE

${ }^{2}$ Service d'Hématologie et Oncologie Pédiatrique, Hopital pour enfants de La Timone, AP-HM. Metronomics Global Health Initiative, Marseille, France

${ }^{3}$ INRIA Paris-Rocquencourt, Domaine de Voluceau, Rocquencourt, B.P. 105, F-78153 Le Chesnay Cedex, France and Université Pierre et Marie Curie, CNRS, UMR 7598, Laboratoire Jacques-Louis Lions, 4, place Jussieu, F-75005, Paris, France

${ }^{4}$ LATP UMR 7353, Aix-Marseille Université, 39 rue F. Joliot Curie, 13453 Marseille cedex 13, FRANCE

${ }^{5}$ ENS Lyon, 46 Allée d'Italie 69007 Lyon, FRANCE

(C) EDP Sciences, SMAI 2013 
thus requires prolonged breaks (generally of 2-3 weeks in duration) between successive cycles to allow recovery from the harmful side effects but also time for cancer tissue to eventually resume growth. Moreover, in the long term, MTD efficacy can be hampered by the propensity of cancer cells to develop resistance to the drug. Hence, toxicity as well as the possible emergence of resistance are main disappointing issues with such chemotherapeutic schedule. In a search for new targets as well as new strategies to fight cancer, Judah Folkman's works on tumor angiogenesis at the beginning of the 70's brought outstanding biological knowledge about tumor biology and initiated a pioneer switch in treatment thinking. Indeed, these works showed that angiogenesis, the process of new blood vessel formation, plays a central role in both local tumor growth and distant metastasis [23, 24]. Therefore, it has become increasingly evident that agents which interfere with blood vessel formation also block tumor progression. Although promising results were initially obtained with such anti-angiogenic treatments, clinical data suggest resistance to these drugs is a very real problem. A new alternative arose in 1991 when R. Kerbel suggested that anti-cancer agents may be able to target tumor vasculature [37]. Then, Klement et al. [39] and mainly Browder et al. [11] showed that a more frequent, regular and lower-dose therapy, administered at one-third of the MTD, had highly anti-angiogenic and anti-tumor effects on several mouse tumor cell lines. This kind of approach was then termed metronomic by Hanahan et al. [31]. Such a protocol, that consists in the frequent, even daily, administration of chemotherapeutics at doses significantly below the MTD, with no prolonged drug-free breaks, seems to inhibit tumor growth primarily through anti-angiogenic mechanisms while significantly reducing undesirable toxic side effects. Both, resistant or not, tumor cells eventually get killed [1,52]. Recent findings suggest that metronomic chemotherapy might also restore anti-cancer immune response and induce tumor dormancy [52]. Nowadays, there is real clinical needs for defining the best schedule of drug administration and related parameters (doses of agent, choice in combination, treatment duration...).

Resorting to mathematical modeling appears as a good way to help clinicians to design optimal administration schedules, for instance by generating hypotheses to explain the effect of metronomic chemotherapies on the vascular network and by performing optimization numerical simulations.

\section{Mathematical models.}

Efficient cancer therapy requires a throughout and upgraded understanding of cancer biochemistry, pharmacology, kinetics and clinics. By using mathematical models, numerous authors have attempted for several years to manage all the available information in order to optimize cancer therapy ( [28]). To optimize drug efficacy, both drug amounts (intensification) and administration schedule (densification) should be determined to ensure a desired rate of tumor cell kill with acceptable toxicity. Twenty years ago, the group of D. Barbolosi and A. Illiadis developed a mathematical model able to calculate densified administration protocols [3,34]. Their approach has been validated through two pioneer works; in [44] they illustrate how such approach may assist to design a phase I trial in oncology, in [58] they report a phase I clinical trial including twenty patients with metastatic breast cancer whose treatment were driven/optimized by a mathematical model. The model consists in a Gompertz model with a death term that represented the cytotoxic action of the chemotherapeutic agent. Pharmacokinetic (PK) and pharmacodynamic (PD) models are used for the cytotoxic action of the chemotherapeutic agents on the cancer cells and for the hematological toxicities. We also can mention few optimization problems analyzed to determine optimal drug infusion schedules taking into account drug toxicity $[6,7,20,40,48-50,56]$. Note that these articles studied the optimization of one anti-cancer therapy (chemotherapy, anti-angiogenic therapy, ...) or of combination of several ones. In order to be more "realistic" such optimization problems can not rely on simple Gompertz law for tumor growth and require more sophisticated model taking into account angiogenic process. Among the models of angiogenesis, let us mention the pioneer works made by the group of J. Folkman $[23,24,29,30]$ since the beginning of the seventies. Several complex models of angiogenesis were then developed, for instance in $[2,12-17,38,51,53]$. Such angiogenesis models were also coupled to tumor growth models in order to study the tumor vascular growth stage, for instance in $[8,10,12,19,33,51,55,60]$. In [8], to study the effects of an anti-angiogenic therapy on tumor growth, the authors coupled, via the concentration of oxygen, the avascular tumor growth model developed by Bresch et al. in [9] with a new multiscale PDE model of angiogenesis. This angiogenesis model accounts for endothelial cell (cells that constitute blood vessel inner wall) density and for pro- and anti-angiogenic agent concentrations. 
Endothelial cells were assumed to be in two different states, that can be named active and non active, according to their ability to respond to pro-angiogenic signals emitted by quiescent tumor cells. Thus active endothelial cells were assumed to proliferate and migrate in the direction of the gradient of pro-angiogenic concentration, i.e. towards the tumor mass, non active endothelial cells were supposed not to proliferate nor migrate but to constitute the wall of efficient blood vessels, source of oxygen. Active endothelial cells were assumed to be able to become non active whereas the inverse was assumed to be impossible, i.e. non active endothelial cells could not become active. By means of this model, Billy et al. analyzed the effects on the vascular network, and so on tumor growth, of several treatment schedules of an anti-angiogenic therapy consisting in promoting the secretion of an anti-angiogenic agent by tumor cells.

Few works exist on the metronomic schedules modeling. Hahnfeldt et al. [29] developed in 2003 a two population ODE model to compare the efficiency of "conventional" and metronomic chemotherapies. The authors considered two cancer cells populations differing by their sensitivity to chemotherapy. Cells of these populations were supposed to proliferate, to gain or loose sensitivity by transiting from one population to the other, and to die due to chemotherapeutic action. Hahnfeldt et al. analyzed several perfusion schedules including a metronomic one and concluded that this one led to better decrease of the tumor size than the other ones.

Majumder and co-workers [42,43,45] compared the effects of metronomic chemotherapy to conventional chemotherapy infusion schedules by means of discrete models. For instance, in [42] the authors developed a discrete model (defined on a point grid) that includes the tumor system, the microvasculature system, and the nearby blood vasculature system. This model also takes into account a tumor angiogenic factor and fibronectin that are known to guide the endothelial cell motion. At each point of the grid and for each time step, the microvascular cell diameter is given by a discrete equation that depends on the probabilities of an individual endothelial cell to move on the nearby points of the grid. The time evolution of the tumor mass was supposed to depend on the microvascular cell diameter at the tumor site. This model enabled to compare the conventional maximum tolerable dose (MTD) strategy with the metronomic one. The authors concluded that metronomic chemotherapy was more efficient to control tumor growth than the maximum tolerable dose chemotherapeutic strategy. In [45], the authors considered two types of tumor cells, i.e. resistant and sensitive to the drug, and the clearance rate of the excretory system that is known to influence the drug concentration and so to influence its toxicity.

The impact of metronomic chemotherapy and conventional chemotherapy on both metastatic and primary tumors, is investigated in [5] by means of a structured transport equation endowed with non local boundary condition. The tumor growth speed, they used was inspired by [30].

For an unresectable glioblastoma, a phenomelogical mathematical model is proposed in [22] able to take into account a classical MTD (Maximum Tolerated Dose) chemotherapy regimen (whose primary targets are the tumor cells) as well as a metronomic chemotherapy regimen (whose primary targets are the tumor endothelial cells) for the administration of Temozolomide (Temodal) in order to compare the effectiveness of these two types of protocols. The model is built from 5 natural hypotheses: without treatment the tumor growth follows a Gompertz model, endothelial cells are more sensitive to temozolomide than cancer cells, the anti-angiogenic effect blocks tumor growth, endothelial cells are genetically stable and thus don't develop resistance to temozolomide, the main chemotherapeutic is due to drug resistance; thus in particular this model of tumor growth take into account this resistance.

As far as the resistance problem is concerned, let us mention the works of [26, 35, 36, 57]. For instance, Jackson et al. [36] developed a PDE model that describes the chemotherapeutic response of a sphericallysymmetric tumor composed of two types of cells differing by their drug sensitivity. The tumor vasculature is indirectly taken into account through the prescribed blood concentration of drug and through the spacedependent rate of blood-tissue transfer of this drug. The authors analyzed the effect of several drug infusion schemes (bolus vs continuous infusion) on the two tumor cell populations. They also analyzed the effect of the vascular distribution on the tumor chemotherapeutic response. Later, Jackson et al. [35] extended this study to asymmetric tumors and included into the model a more mechanistic modeling of the tumor vasculature by considering the density of effective vessels as a dynamic variable of the model. 
Outline. We present a unified continuous PDE model, able to handle both conventional chemotherapies with their problems of resistance and metronomic schedule with their anti-angiogenic effect. This model, derived from [8] study the combined dynamics of proliferant tumor cells, quiescent tumor cells, dead cells, normal cells with the endothelial cells (active or non active). The influence of oxygen, angiogenic factors and chemotherapy drugs on these cells is investigated.

This paper is organized as follows: Section 2 presents the biological assumptions we made and the resulting equations; rules that have to be respected by the model are summarized in paragraph 2.4; finally the numerical illustrations constitute Section 3 and the conclusion stands in Section 4 together with a discussion.

\section{Construction of the model}

\subsection{Biological hypotheses}

At first, the tumor cells divide continuously, we call them proliferating and the resulting tumor consumes nutrients and oxygen brought by the blood and lymph pre-existing systems. During this phase, termed avascular, tumor is off a few millimeters and is rarely detected by medical imaging. When it reaches a diameter of $2-3$ mm, nutrient intakes and oxygen become insufficient so that a portion of proliferating tumor cells stop their division and enter in a quiescent state. This is called hypoxia when oxygen tension [pO2] is less than 2.5mmHg. Note that quiescent tumor cells may die if hypoxia is to high. For further growth, the tumor stimulates endothelial cells (ECs) forming the walls surrounding blood vessels, through secretion of "pro-angiogenic" factors, foremost among which VEGF to develop neovascularization. These compete with those so-called "anti-angiogenic" factors naturally present in the body and secreted in response to the abnormal presence of pro-angiogenic factors. These factors take over, active the division of endothelial cells that migrate to the area of secretion of VEGF to form the neo-vasculature. These new blood vessels differ from the physiological vasculature; they are dense and irregular, some of them may be non-active. Therefore quiescent tumor cells within the tumor may become again proliferating as soon as the concentration of oxygen is again sufficient.

Non active ECs proliferate and become active when the number of ECs point is large enough. Tumor cells as well as dead cells can destroy ECs in the neighborhood of the tumor (hence a potential decline in the supply of O2). Dead cells are gradually eliminated by macrophages arriving via the blood vessels.

The chemotherapy is a cytotoxic agent supposed to kill all proliferating cells (tumor cells and endothelial

cells). In contrast to ECs, tumor cells can develop resistance to chemotherapy, depending on the amount of drug already administered (cumulative drug dose).

\subsection{Equations}

All the variables of the model depend on time $t$ and space $x \in \mathbb{R}^{d}$ with $d=2$ or $d=3$.

The variables appearing in the model are directly linked to the biological entities we considered, i.e.

$\mathbf{o}_{2}$ : Tissue relative concentration (in $[0 ; 1]$ ) of oxygen.

$\mathbf{a}_{n g}$ : Angiogenic balance between pro- $(\mathrm{VEGF}, \ldots)$ and anti- (angiostatin, ...) angiogenic factors.

$\mathbf{e c}_{a}$ : Active EC density.

$\mathbf{e c}_{n a}$ : Non active EC density.

p: Proliferant tumor cell density.

q: Quiescent tumor cell density.

m: Viable healthy cell density.

d: Dead cell density coming from the tumor as well as the healthy tissue.

$v$ : Velocity of cell moving linked to cell proliferation.

$p$ : Pressure linked to cell proliferation.

$C$ : Chemotherapeutic agent (cytotoxic) pulsed concentration.

Figure 1 summarizes the principle of the dynamics. 


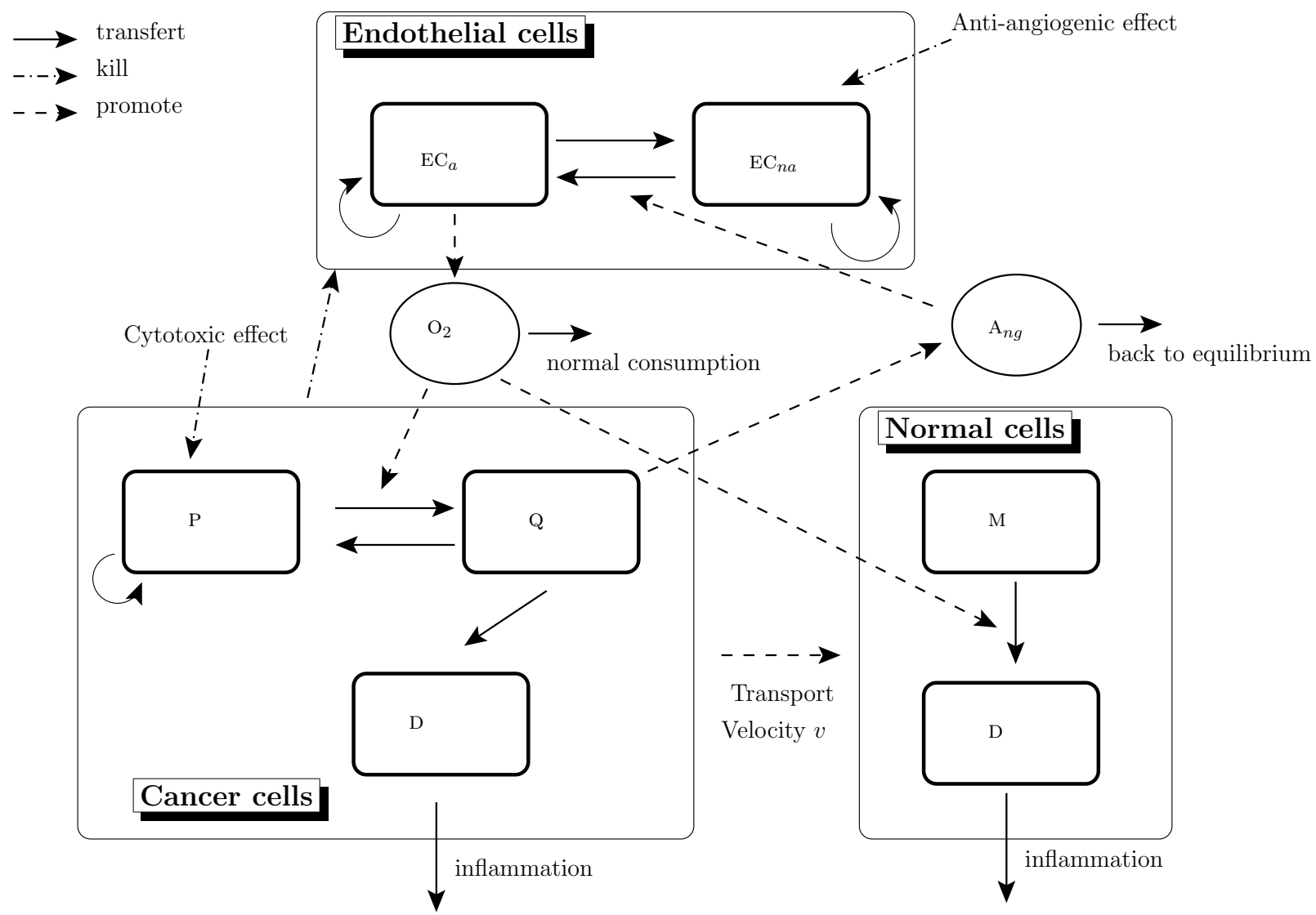

Figure 1. The model principle. The dynamics of cancer cells is linked to the presence of nutrients (oxygen). In case of hypoxia, they activate angiogenic factors. These angiogenic factors act on the dynamics of endothelial cells. These endothelial cells in turn act on the dynamics of oxygen. The growth of cancer cells induces finally a transport of both cancer cells and normal cells.

Oxygen. Oxygen is supposed to diffuse in the surrounding tissue from the functional blood vessels represented by active ECs. It is also assumed to disappear because of natural degradation. The cell consumption is neglected and the concentration is maintained at a maximal fixed value $\mathrm{O}_{2}^{\max }$ in the active blood vessel. We assume that the time scale associated to oxygen diffusion is very small compared to the one of cell division, so that the oxygen dynamics is governed by a quasi-static equation:

$$
0=D_{\mathrm{O}} \Delta \mathrm{O}_{2}-\delta_{\mathrm{O}} \mathrm{O}_{2}+\delta_{\mathrm{O}} \mathrm{O}_{2}^{\max } \mathrm{EC}_{a}
$$

where $D_{\mathrm{O}}$ is the diffusion rate, $\delta_{\mathrm{O}}$ the degradation rate. The quantities $D_{\mathrm{O}}, \mathrm{O}_{2}^{\max }$ and $\delta_{\mathrm{O}}$ are assumed to be constant and positive.

Angiogenic balance. The angiogenic balance resumes the effects of pro- and anti- angiogenic factors. We supposed that they diffuse in the tissue. They are produced by quiescent tumor cells at a constant rate, and are naturally eliminated. As we did with oxygen, we assumed that the dynamics of angiogenic factors is also governed by a quasi-static equation:

$$
0=D_{\mathrm{v}} \Delta \mathrm{A}_{n g}+\alpha_{\mathrm{V}} \mathrm{Q}-\delta_{\mathrm{V}} \mathrm{A}_{n g},
$$


where $D_{\mathrm{V}}$ is the diffusion rate, $\alpha_{\mathrm{V}}$ the excretion rate by quiescent tumor cells $\mathrm{Q}, \delta_{\mathrm{V}}$ the degradation rate. The quantities $D_{\mathrm{V}}, \alpha_{\mathrm{V}}$ and $\delta_{\mathrm{V}}$ are assumed to be constant and positive.

Active ECs. Active ECs are assumed to diffuse in the tissue. Part of them may become non active as soon as angiogenic factors reached a given threshold $s_{\mathrm{A}_{n g}, \mathrm{EC}}$. Non active ECs are supposed to stabilize, i.e. to become active as soon as the total EC density was higher than a given threshold $\mathrm{EC}_{\min }$. These cells are also supposed to disappear due to necrotic and tumor cells local dominance. Active ECs are to recolonize slowly the space in case of low ECs density. Thus, the evolution of the active EC density is given by:

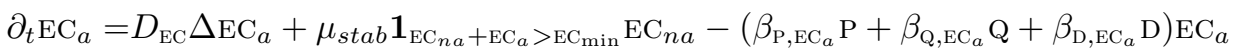

$$
\begin{aligned}
& -\mu_{i n s t} \operatorname{Sig}\left(\mathrm{A}_{n g}, r_{\mathrm{A}_{n g}, \mathrm{EC}}, s_{\mathrm{A}_{n g}, \mathrm{EC}}\right) \mathrm{EC}_{a}+\alpha_{r e c o l} \mathrm{EC}_{a}\left(1-\left(\mathrm{EC}_{n a}+\mathrm{EC}_{a}\right)\right) \text {, }
\end{aligned}
$$

where $D_{\mathrm{EC}}$ represents the diffusion coefficient, $\mu_{s t a b}$ the constant transition rate from non active to active ECs, $\beta_{\mathrm{P}, \mathrm{EC}_{a}}, \beta_{\mathrm{Q}_{\mathrm{EC} a}}, \beta_{\mathrm{D}, \mathrm{EC}_{a}}$ the elimination rates due to necrotic and tumor cell local dominance, $\alpha_{\text {recol }}$ the proliferation rate, $\mu_{i n s t} \operatorname{Sig}\left(\mathrm{A}_{n g}, r_{\mathrm{A}_{n g}, \mathrm{EC}}, s_{\mathrm{A}_{n g}, \mathrm{EC}}\right)$ the transition rate from non active to active ECs where $\mu_{i n s t}$ is assumed to be constant, and where the function $\operatorname{Sig}$ is defined by: $\operatorname{Sig}(w, y, z)=\frac{w}{1+e^{-y(w-z)}}$

Non active ECs. As said before, we assume that non active ECs derived from active ECs as soon as angiogenic factors reach a threshold $s_{\mathrm{A}_{n g}, \mathrm{EC}}$. They begin to proliferate until the total EC density reach a maximal value $\mathrm{EC}_{\max }=1$. Non active EC loss is supposed to be induced by stabilization into active ECs, by elimination due to necrotic and tumor cell local presence and by the cytotoxic effect of the chemotherapy. Thus non active EC density follows the equation:

$$
\begin{aligned}
& \partial_{t} \mathrm{EC}_{n a}=\alpha_{\mathrm{EC}} \mathbf{1}_{\mathrm{A}_{n g}>s_{\mathrm{A}_{n g}, \mathrm{EC}}} \mathrm{EC}_{n a}\left(1-\left(\mathrm{EC}_{n a}+\mathrm{EC}_{a}\right)\right)+\mu_{i n s t} \operatorname{Sig}\left(\mathrm{A}_{n g}, r_{\mathrm{A}_{n g}, \mathrm{EC}}, s_{\mathrm{A}_{n g}, \mathrm{EC}}\right) \mathrm{EC}_{a} \\
& -\mu_{s t a b} \mathbf{1}_{\mathrm{EC}_{n a}+\mathrm{EC}_{a}>\mathrm{EC}_{\min }} \mathrm{EC}_{n a}-\left(\delta_{\mathrm{EC}}+\beta_{\mathrm{P}_{, \mathrm{EC}_{n a}} \mathrm{P}}+\beta_{\mathrm{Q}, \mathrm{EC}_{n a}} \mathrm{Q}+\beta_{\mathrm{D}_{\mathrm{EEC} n a}} \mathrm{D}\right) \mathrm{EC}_{n a} \\
& -\eta_{\mathrm{EC}}\left(C(t)-\mathrm{C}_{\min , \mathrm{AA}}\right)^{+} \mathrm{EC}_{n a}
\end{aligned}
$$

where $\alpha_{\mathrm{EC}}$ represents the proliferation rate, $\mu_{\text {inst }}$ the destabilization rate, $\mu_{\text {stab }}$ the stabilization rate, $\beta_{\mathrm{P}, \mathrm{EC}_{n a}}$, $\beta_{\mathrm{Q}, \mathrm{EC}_{n a}}$ and $\beta_{\mathrm{D}, \mathrm{EC}_{n a}}$ the loss rates due to tumor cell presence, $\delta_{\mathrm{EC}}$ the natural decay rate and $\eta_{\mathrm{EC}}$ the cytotoxic effect rate. The function Sig is previously defined.

Note that for both ECs, the migration under growing tumor pressure has been neglected.

Tumor cells. We distinguish among the tumor cells, the proliferant and the quiescent cells. Proliferant cells divide with a constant rate $\alpha_{\mathrm{P}}$ and are supposed to become quiescent if the oxygen level falls below a given threshold $h_{\mathrm{PQ}}$. Quiescent tumor cells are supposed to become proliferant if the oxygen level exceeds a given threshold $h_{\mathrm{QP}}$ or to die of the oxygen level falls below a given threshold $h_{\mathrm{QD}}$. Tumor cell proliferation and death induce a passive transport movement whose velocity is denoted by $v$ (cf. below for more details about $v$ ). Proliferant tumor cells, as well as all proliferant cells, are also supposed to be killed by the cytotoxic effect of the chemotherapy. So the evolution of the proliferant and quiescent tumor cell densities is given by:

$$
\begin{aligned}
& \partial_{t} \mathrm{P}+\nabla \cdot(v \mathrm{P})=\alpha_{\mathrm{P}} \mathrm{P}-\gamma_{\mathrm{PQ}} f_{-}\left(\mathrm{O}_{2}, h_{\mathrm{PQ}}\right) \mathrm{P}+\gamma_{\mathrm{QP}} f_{+}\left(\mathrm{O}_{2}, h_{\mathrm{QP}}\right) \mathrm{Q}-\eta_{\mathrm{P}} e^{-\alpha_{r} \operatorname{expo}(t)}\left(C(t)-\mathrm{C}_{\mathrm{min}, \mathrm{CT}}\right)^{+} \mathrm{P}, \\
& \partial_{t} \mathrm{Q}+\nabla \cdot(v \mathrm{Q})=\gamma_{\mathrm{PQ}} f_{-}\left(\mathrm{O}_{2}, h_{\mathrm{PQ}}\right) \mathrm{P}-\gamma_{\mathrm{QP}} f_{+}\left(\mathrm{O}_{2}, h_{\mathrm{QP}}\right) \mathrm{Q}-\gamma_{\mathrm{QD}} f_{-}\left(\mathrm{O}_{2}, h_{\mathrm{QD}}\right) \mathrm{Q},
\end{aligned}
$$

where $x^{+}=\frac{1}{2}(x+|x|), f_{-}(o, h)=\mathbb{1}_{o<h}\left(1-\frac{o}{h}\right)$, and $f_{+}(o, h)=\mathbb{1}_{o>h}\left(\frac{o-h}{O_{2}^{\max }-h}\right)$. The positive constants $\gamma_{\mathrm{PQ}}, \gamma_{\mathrm{QP}}, \gamma_{\mathrm{Q}, \mathrm{D}}$ are elimination rates. The chemotherapy's action will be detailed further.

Viable healthy cells. Viable healthy cells are transported under the velocity $v$ induced by the tumor growth. We assume that healthy cells are eliminated in case of shortage of oxygen. Their equation reads:

$$
\partial_{t} \mathrm{M}+\nabla \cdot(v \mathrm{M})=-\gamma_{\mathrm{MD}} f_{-}\left(\mathrm{O}_{2}, h_{\mathrm{MD}}\right) \mathrm{M}
$$

where $f_{-}$is defined above and $h_{\mathrm{MD}}$ stands for the oxygen threshold, $\gamma_{\mathrm{MD}}$ is a degradation rate. 


\section{Necrotic / apoptotic tumor and healthy cells.}

Necrotic cells are supposed to result from quiescent cells and healthy cells if the oxygen level falls below some threshold $h_{\mathrm{QD}}$ and $h_{\mathrm{MD}}$. They are gradually eliminated by macrophages due to inflammatory processes: we assume that these macrophages diffused from the surrounded functional blood vessels so we suppose that their concentration was proportional to the oxygen concentration. Necrotic cells are also supposed to be transported with velocity $v$ by the growing tumor mass. So the evolution of the density of necrotic cells is given by:

$$
\partial_{t} \mathrm{D}+\nabla \cdot(v \mathrm{D})=\gamma_{\mathrm{QD}} f_{-}\left(\mathrm{O}_{d}, h_{\mathrm{QD}}\right) \mathrm{Q}+\gamma_{\mathrm{MD}} f_{-}\left(\mathrm{O}_{2}, h_{\mathrm{MD}}\right) \mathrm{M}-\delta_{\mathrm{infl}} \mathrm{O}_{2} \mathrm{D}
$$

where $f_{-}$is defined above and where $\delta_{\text {infl }}$ represented the degradation rate due to macrophages.

Velocity. We assume that the cell movement due to the growing tumor mass follows a Darcy's law:

$$
v=-\nabla p,
$$

where $p$ is the pressure induced by the tumor growth. The velocity is supposed to be independant of the type of cells.

To prescribe an expression for the pressure $p$, we assumed that the total number of tumor, necrotic and healthy cells $P+Q+D+M$ is constant (equal to 1) with respect to time when no chemotherapy is applied. So by summing equations (5) to (8), and using Darcy's law, we get:

$$
-\Delta p=\nabla \cdot v=\alpha_{\mathrm{P}} \mathrm{P}-\delta_{\text {infl }} \mathrm{O}_{2} \mathrm{D}-\eta_{\mathrm{P}} e^{-\alpha_{r} \operatorname{expo}(t)}\left(C(t)-\mathrm{C}_{\min , \mathrm{CT}}\right)^{+} \mathrm{P}
$$

Chemotherapeutic agent. The chemotherapy acts on any proliferating cells, that is here on both proliferating tumor cells and non active ECs. We first assume that the concentration of the drug has to reach a threshold $\mathrm{C}_{\min , \mathrm{CT}}$ to be efficient on the proliferating tumor cells and a threshold $\mathrm{C}_{\min , \mathrm{AA}}$ on endothelial cells. As tumor cells tend to develop drug resistance, we assumed that this drug response was exponentially decreasing according to the total drug exposure $\operatorname{expo}(t)=\int_{0}^{t} C(\tau) d \tau$. Remark that endothelial cells are genetically more stable and thus much less likely to become resistant.

The model. The model equations are summarized below.

$$
\begin{aligned}
& 0=D_{\mathrm{O}} \Delta \mathrm{O}_{2}-\delta_{\mathrm{O}} \mathrm{O}_{2}+\delta_{\mathrm{O}} \mathrm{O}_{2}^{\max } \mathrm{EC}_{a} \\
& 0=D_{\mathrm{V}} \Delta \mathrm{A}_{n g}+\alpha_{\mathrm{V}} \mathrm{Q}-\delta_{\mathrm{V}} \mathrm{A}_{n g}
\end{aligned}
$$

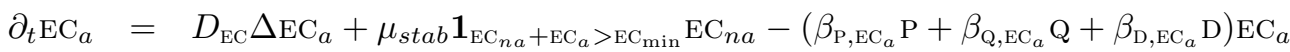

$$
\begin{aligned}
& -\mu_{i n s t} \operatorname{Sig}\left(\mathrm{A}_{n g}, r_{\mathrm{A}_{n g}, \mathrm{EC}}, s_{\mathrm{A}_{n g}, \mathrm{EC}}\right) \mathrm{EC}_{a}+\alpha_{r e c o l} \mathrm{EC}_{a}\left(1-\left(\mathrm{EC}_{n a}+\mathrm{EC}_{a}\right)\right) \\
& \partial_{t} \mathrm{EC}_{n a}=\alpha_{\mathrm{EC}} \mathbf{1}_{\mathrm{A}_{n g}>s_{\mathrm{A}_{n}, \mathrm{EC}}} \mathrm{EC}_{n a}\left(1-\left(\mathrm{EC}_{n a}+\mathrm{EC}_{a}\right)\right)+\mu_{i n s t} S i g\left(\mathrm{~A}_{n g}, r_{\mathrm{A}_{n g}, \mathrm{EC}}, s_{\mathrm{A}_{n g}, \mathrm{EC}}\right) \mathrm{EC}_{a} \\
& -\mu_{s t a b} \mathbf{1}_{\mathrm{EC}_{n a}+\mathrm{EC}_{a}>\mathrm{EC}_{\min }} \mathrm{EC}_{n a}-\left(\delta_{\mathrm{EC}}+\beta_{\mathrm{P}_{, \mathrm{EC}_{n a}} \mathrm{P}}+\beta_{\mathrm{Q}_{\mathrm{ECC} n a} \mathrm{Q}}+\beta_{\mathrm{D}, \mathrm{EC}_{n a}} \mathrm{D}\right) \mathrm{EC}_{n a} \\
& -\eta_{\mathrm{EC}}\left(C(t)-\mathrm{C}_{\min , \mathrm{AA}}\right)^{+} \mathrm{EC}_{n a} \\
& \partial_{t} \mathrm{P}+\nabla \cdot(v \mathrm{P})=\alpha_{\mathrm{P}} \mathrm{P}-\gamma_{\mathrm{PQ}} f_{-}\left(\mathrm{O}_{2}, h_{\mathrm{PQ}}\right) \mathrm{P}+\gamma_{\mathrm{QP}} f_{+}\left(\mathrm{O}_{2}, h_{\mathrm{QP}}\right) \mathrm{Q}-\eta_{\mathrm{P}} e^{-\alpha_{r} \operatorname{expo}(t)}\left(C(t)-\mathrm{C}_{\mathrm{min}, \mathrm{CT}}\right)^{+} \mathrm{P} \\
& \partial_{t} \mathrm{Q}+\nabla \cdot(v \mathrm{Q})=\gamma_{\mathrm{PQ}} f_{-}\left(\mathrm{O}_{2}, h_{\mathrm{PQ}}\right) \mathrm{P}-\gamma_{\mathrm{QP}} f_{+}\left(\mathrm{O}_{2}, h_{\mathrm{QP}}\right) \mathrm{Q}-\gamma_{\mathrm{QD}} f_{-}\left(\mathrm{O}_{2}, h_{\mathrm{QD}}\right) \mathrm{Q} \\
& \partial_{t} \mathrm{M}+\nabla \cdot(v \mathrm{M})=-\gamma_{\mathrm{MD}} f_{-}\left(\mathrm{O}_{2}, h_{\mathrm{MD}}\right) \mathrm{M} \\
& \partial_{t} \mathrm{D}+\nabla \cdot(v \mathrm{D})=\gamma_{\mathrm{QD}} f_{-}\left(\mathrm{O}_{2}, h_{\mathrm{QD}}\right) \mathrm{Q}+\gamma_{\mathrm{MD}} f_{-}\left(\mathrm{O}_{2}, h_{\mathrm{MD}}\right) \mathrm{M}-\delta_{\mathrm{infl}_{2} \mathrm{O}_{2} \mathrm{D}} \\
& v=-\nabla p \\
& -\Delta p=\nabla \cdot v=\alpha_{\mathrm{P}} \mathrm{P}-\delta_{\text {infl }} \mathrm{O}_{2} \mathrm{D}-\eta_{\mathrm{P}} e^{-\alpha_{r} \operatorname{expo}(t)}\left(C(t)-\mathrm{C}_{\mathrm{min}, \mathrm{CT}}\right)^{+} \mathrm{P}
\end{aligned}
$$

where $\operatorname{Sig}(w, y, z)=\frac{w}{1+e^{-y(w-z)}}, f_{-}(o, h)=\mathbb{1}_{o<h}\left(1-\frac{o}{h}\right), f_{+}(o, h)=\mathbb{1}_{o>h}\left(\frac{o-h}{\mathrm{o}_{2}^{\text {max }}-h}\right)$ et expo $(t)=\int_{0}^{t} C(\tau) d \tau$ (see Figure 2). 

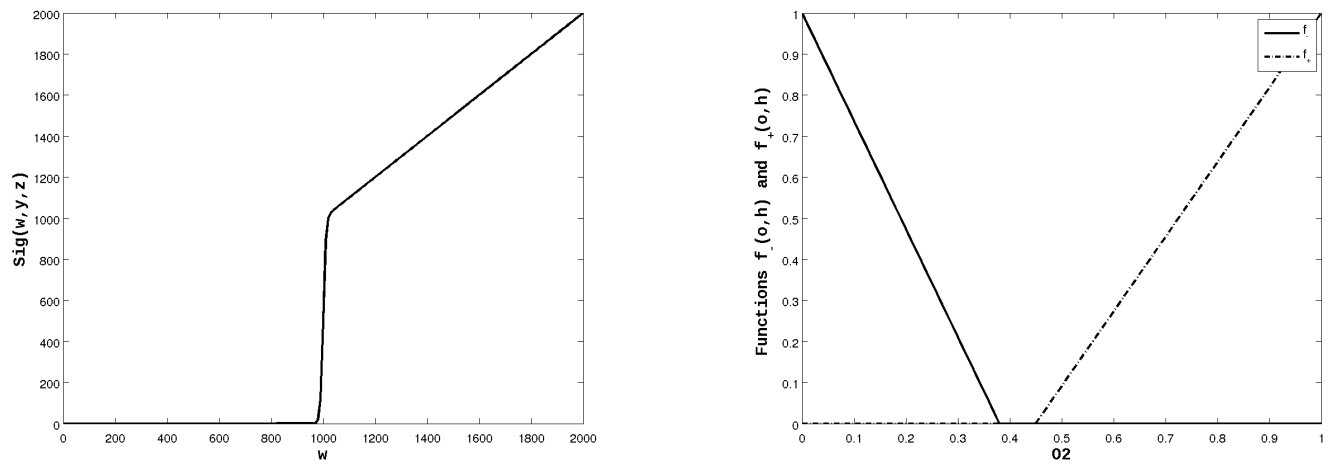

Figure 2. (Left). Graph of the function $w \mapsto \operatorname{Sig}(w, y, z)$ with $y=r_{\mathrm{A}_{n g}, \mathrm{EC}}$ and $z=s_{\mathrm{A}_{n g}, \mathrm{EC}}$. (Right). Graph of the functions $o \mapsto f_{-}(o, h)$ with $h=h_{\mathrm{PQ}}$ and $o \mapsto f_{+}(o, h)$ with $h=h_{\mathrm{QP}}$.

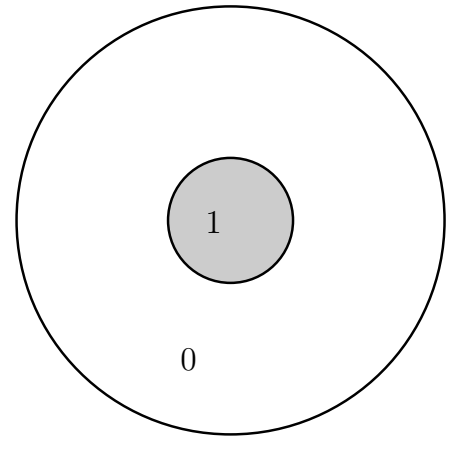

$\mathrm{P}$ at $t=0$

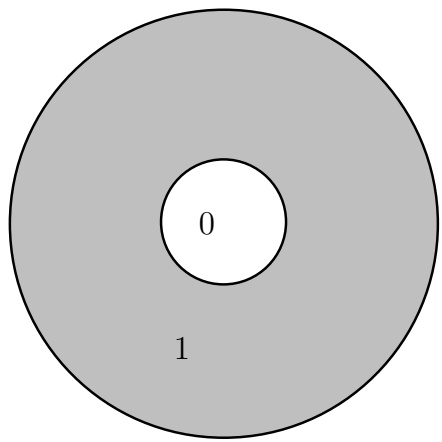

$\mathrm{M}, \mathrm{EC}_{a}$ at $t=0$

Figure 3. Radial representation of initial data $\mathrm{P}, \mathrm{M}$ and $\mathrm{EC}_{a}$ for the various simulations. Distances are in millimeters. The tumor is located at the center of the domain and has an initial radius of $0.1 \mathrm{~mm}$

Boundary conditions. The computational domain is assumed to be large enough to avoid boundary effects. We assumed homogeneous Dirichlet boundary conditions, except for $\mathrm{O}_{2}$ and $\mathrm{M}$ that were supposed to respectively satisfy $\mathrm{O}_{2}=\mathrm{O}_{2}^{\max }$ and $\mathrm{M}=1$ on the domain boundaries.

Initial conditions. We restrict in this paper to radially initial conditions (at time $t=0$ ):

$$
\mathrm{EC}_{n a}=0, \mathrm{EC}_{a}=\mathbb{1}_{x \notin \text { tumor }}, \mathrm{P}=\mathbb{1}_{x \in \text { tumor }}, \mathrm{Q}=0, \mathrm{D}=0, \mathrm{M}=\mathbb{1}_{x \notin \text { tumor }}
$$

where tumor corresponds to the initial tumor location, i.e., a circle (in 2D) or a sphere (in 3D) centered on the space location $(0,0)$ (in 2D), $(0,0,0)$ (in 3D), whose radius is equal to $r_{\text {tumor }}$ (see figure 3 ).

\subsection{Discretization scheme}

The assumption on the polar/spherical initial location of the tumor, implies a polar/spherical symmetry of the solution of the model. All the scalar quantities $Y$ are thus functions $Y(t, r)$ of the time and the radius $r$ of the tumor and the velocity $v=v(t, r)$ is radially oriented. The new domain of calculation is $[0, T] \times[0, R]$, and 
the new set of equations just consists in replacing the two operators $\Delta$ and $\nabla \cdot$ by

$$
\begin{aligned}
\Delta & \leftrightarrow \quad \Delta_{r}:=\frac{1}{r^{d-1}} \partial_{r}\left(r^{d-1} \partial_{r} \cdot\right) \text { with } d \text { the space dimension } \\
\nabla \cdot & \leftrightarrow \quad(\nabla \cdot)_{r}:=\frac{1}{r^{d-1}} \partial_{r}\left(r^{d-1} \cdot\right) .
\end{aligned}
$$

Let $d t=\frac{1}{N}$ denote the time step; the time interval $[0, T]$ is uniformly discretized by $t_{n}=n d t$ for $n=0, \cdots, N$. Let $h=\frac{1}{M+1}$ the space step; the interval $[0, R]=\cup_{i=1}^{M} J_{i}$ is split into $M$ sub-interval $J_{i}=\left[r_{i-\frac{1}{2}}, r_{i+\frac{1}{2}}\right]$ with $r_{i+\frac{1}{2}}=i h$. Let $r_{i}=\frac{1}{2}\left(r_{i-\frac{1}{2}}+r_{i+\frac{1}{2}}\right)=\left(i-\frac{1}{2}\right) h$ be the center of the intervals $J_{i}$. All the functions $Y(t, r)$ are approximated at $\left(t_{n}, r_{i}\right)$ by a quantity $Y_{i}^{n}$. Let $Y^{n}$ be the vector $Y^{n}=\left(Y_{i}^{n}\right)_{i=1, \cdots, M}$.

We use a semi-implicit Euler scheme for the time discretization. The terms treated implicitely are specified below:

$$
\begin{aligned}
& 0=D_{\mathrm{O}} \Delta_{r}^{h} \mathrm{O}_{2}^{n+1}-\delta_{\mathrm{O}} \mathrm{O}_{2}^{n+1}+\delta_{\mathrm{O}} \mathrm{O}_{2}^{\max } \mathrm{EC}_{a}^{n} \\
& 0=D_{\mathrm{V}} \Delta_{r}^{h} \mathrm{~A}_{n g}^{n+1}+\alpha_{\mathrm{V}} \mathrm{Q}^{n}-\delta_{\mathrm{V}} \mathrm{A}_{n g}^{n+1} \\
& -\Delta_{r}^{h} p^{n+1}=\alpha_{\mathrm{P}} \mathrm{P}^{n}-\delta_{i n f l} \mathrm{O}_{2}^{n+1} \mathrm{D}^{n}-\eta_{\mathrm{P}} e^{-\alpha_{r} \operatorname{expo}\left(t_{n}\right)}\left(C\left(t_{n}\right)-\mathrm{C}_{\min , \mathrm{CT}}\right)^{+} \mathrm{P}^{n} \\
& v^{n+1}=-\nabla_{r}^{h} p^{n+1} \\
& \frac{1}{d t}\left(\mathrm{EC}_{n a}^{n+1}-\mathrm{EC}_{n a}^{n}\right)=\alpha_{\mathrm{EC}} \mathbf{1}_{\mathrm{A}_{n g}^{n+1}>s_{\mathrm{A} n g}, \mathrm{EC}} \mathrm{EC}_{n a}^{n+1}\left(1-\left(\mathrm{EC}_{n a}^{n}+\mathrm{EC}_{a}^{n}\right)\right) \\
& +\mu_{i n s t} \operatorname{Sig}\left(\mathrm{A}_{n g}^{n+1}, r_{\mathrm{A}_{n g}, \mathrm{EC}}, s_{\mathrm{A}_{n g}, \mathrm{EC}}\right) \mathrm{EC}_{a}^{n}-\mu_{s t a b} \mathbf{1}_{\mathrm{EC}_{n a}^{n}+\mathrm{EC}_{a}^{n}>\mathrm{EC}_{\min }} \mathrm{EC}_{n a}^{n+1} \\
& -\left(\delta_{\mathrm{EC}}+\beta_{\mathrm{P}, \mathrm{EC}_{n a}} \mathrm{P}^{n}+\beta_{\mathrm{Q}, \mathrm{EC}_{n a}} \mathrm{Q}^{n}+\beta_{\mathrm{D}, \mathrm{EC}_{n a}} \mathrm{D}^{n}\right) \mathrm{EC}_{n a}^{n+1} \\
& -\eta_{\mathrm{EC}}\left(C\left(t_{n}\right)-\mathrm{C}_{\min , \mathrm{AA}}\right)^{+} \mathrm{EC}_{n a}^{n+1} \\
& \frac{1}{d t}\left(\mathrm{EC}_{a}^{n+1}-\mathrm{EC}_{a}^{n}\right)=D_{\mathrm{EC}} \Delta_{r}^{h} \mathrm{EC}_{a}^{n+1}+\mu_{s t a b} \mathbf{1}_{\mathrm{EC}_{n a}^{n}+\mathrm{EC}_{a}^{n}>\mathrm{EC}_{\min }} \mathrm{EC}_{n a}^{n+1} \\
& -\left(\beta_{\mathrm{P}, \mathrm{EC}_{a}} \mathrm{P}^{n}+\beta_{\mathrm{Q}, \mathrm{EC}_{a}} \mathrm{Q}^{n}+\beta_{\mathrm{D}, \mathrm{EC}_{a}} \mathrm{D}^{n}\right) \mathrm{EC}_{a}^{n+1} \\
& -\mu_{i n s t} \operatorname{Sig}\left(\mathrm{A}_{n g}^{n+1}, r_{\mathrm{A}_{n g}, \mathrm{EC}}, s_{\mathrm{A}_{n g}, \mathrm{EC}}\right) \mathrm{EC}_{a}^{n+1} \\
& +\alpha_{\text {recol }} \mathrm{EC}_{a}^{n}\left(1-\left(\mathrm{EC}_{n a}^{n}+\mathrm{EC}_{a}^{n}\right)\right) \\
& \frac{1}{d t}\left(\mathrm{P}^{n+1}-\mathrm{P}^{n}\right)+(\nabla \cdot)_{r}^{h}\left(v \mathrm{P}^{n+1}\right)=\alpha_{\mathrm{P}} \mathrm{P}^{n}-0 \gamma_{\mathrm{PQ}} f_{-}\left(\mathrm{O}_{2}^{n+1}, h_{\mathrm{PQ}}\right) \mathrm{P}^{n+1}+\gamma_{\mathrm{QP}} f_{+}\left(\mathrm{O}_{2}^{n+1}, h_{\mathrm{QP}}\right) \mathrm{Q}^{n} \\
& -\eta_{\mathrm{P}} e^{-\alpha_{r} \operatorname{expo}\left(t_{n}\right)}\left(C\left(t_{n}\right)-\mathrm{C}_{\min , \mathrm{CT}}\right)^{+} \mathrm{P}^{n} \\
& \frac{1}{d t}\left(\mathrm{Q}^{n+1}-\mathrm{Q}^{n}\right)+(\nabla \cdot)_{r}^{h}\left(v \mathrm{Q}^{n+1}\right)=\gamma_{\mathrm{PQ}} f_{-}\left(\mathrm{O}_{2}^{n+1}, h_{\mathrm{PQ}}\right) \mathrm{P}^{n+1}-\gamma_{\mathrm{QP}} f_{+}\left(\mathrm{O}_{2}^{n+1}, h_{\mathrm{QP}}\right) \mathrm{Q}^{n}-\gamma_{\mathrm{QD}} f_{-}\left(\mathrm{O}_{2}^{n+1}, h_{\mathrm{QD}}\right) \mathrm{Q}^{n+1} \\
& \frac{1}{d t}\left(\mathrm{D}^{n+1}-\mathrm{D}^{n}\right)+(\nabla \cdot)_{r}^{h}\left(v \mathrm{D}^{n+1}\right)=\gamma_{\mathrm{QD}} f_{-}\left(\mathrm{O}_{2}^{n+1}, h_{\mathrm{QD}}\right) \mathrm{Q}^{n+1}+\gamma_{\mathrm{MD}} f_{-}\left(\mathrm{O}_{2}^{n+1}, h_{\mathrm{MD}}\right) \mathrm{M}^{n}-\delta_{\mathrm{infl}} \mathrm{O}_{2} \mathrm{D}^{n} \\
& \frac{1}{d t}\left(\mathrm{M}^{n+1}-\mathrm{M}^{n}\right)+(\nabla \cdot)_{r}^{h}\left(v \mathrm{M}^{n+1}\right)=-\gamma_{\mathrm{MD}} f_{-}\left(\mathrm{O}_{2}^{n+1}, h_{\mathrm{MD}}\right) \mathrm{M}^{n}
\end{aligned}
$$

Note that this discretization preserves the mass balance equation $\mathrm{P}+\mathrm{Q}+\mathrm{D}+\mathrm{M}=C t e$, ie for all $n$,

$$
\mathrm{P}^{n}+\mathrm{Q}^{n}+\mathrm{D}^{n}+\mathrm{M}^{n}=1
$$

The finite volume discretization in space (see [21]) consists in multiplying all the equations by $r^{d-1}$, with $d$ being the space dimension, and integrating the resulting equations over $J_{i}$. The diffusion terms

$$
\int_{J_{i}} r^{d-1} \Delta_{r} Y=\left[r^{d-1} \partial_{r} Y\right]_{x_{i-\frac{1}{2}}}^{x_{i+\frac{1}{2}}}
$$


are approximated by $h r_{i}^{d-1} \Delta_{r}^{h} Y$ where for $Y \in \mathbb{R}^{M}$

$$
\begin{aligned}
h r_{1}^{d-1}\left(\Delta_{r}^{h} Y\right)_{1} & =r_{\frac{3}{2}}^{d-1} \frac{Y_{2}-Y_{1}}{h} \\
h r_{i}^{d-1}\left(\Delta_{r}^{h} Y\right)_{i} & =r_{i+\frac{1}{2}}^{d-1} \frac{Y_{i+1}-Y_{i}}{h}-r_{i-\frac{1}{2}}^{d-1} \frac{Y_{i}-Y_{i-1}}{h}, i=2, \cdots, M \\
h r_{M}^{d-1}\left(\Delta_{r}^{h} Y\right)_{M} & =r_{M+\frac{1}{2}}^{d-1} \frac{Y_{M+\frac{1}{2}}-Y_{M}}{\frac{h}{2}}-r_{M-\frac{1}{2}}^{d-1} \frac{Y_{M}-Y_{M-1}}{h},
\end{aligned}
$$

where $Y_{M+\frac{1}{2}}$ is given by the boundary condition at $r=R$.

Note that the velocity $v$ is then naturally approximated at the vertices $r_{i+\frac{1}{2}}$ of the segments $J_{i}$ by $v_{i+\frac{1}{2}}=$ $\frac{1}{h}\left(p_{i+1}-p_{i}\right)$ for $i=1, \cdots, M-1$ and $v_{M+\frac{1}{2}}=\frac{2}{h}\left(0-p_{M}\right)$ using the homogeneous boundary condition on $p$ at $r=R$. The velocity $v_{\frac{1}{2}}$ can be taken equal to zero without loss of generality since it is always multiplied by $r_{\frac{1}{2}}=0$. The transport terms are then approximated thanks to an upwind scheme

$$
\int_{J_{i}} r^{d-1}(\nabla \cdot)_{r}(v Y)=\left[\partial_{r}(v Y)\right]_{x_{i-\frac{1}{2}}}^{x_{i+\frac{1}{2}}} \sim h r_{i}^{d-1}(\nabla \cdot)_{r}^{h}(v Y)
$$

with

$$
\begin{aligned}
h r_{1}^{d-1}\left((\nabla \cdot)_{r}^{h}(v Y)\right)_{1} & =r_{\frac{3}{2}}^{d-1}\left(v_{\frac{3}{2}}^{+} Y_{1}-v_{\frac{3}{2}}^{-} Y_{2}\right) \\
h r_{i}^{d-1}\left((\nabla \cdot)_{r}^{h}(v Y)\right)_{i} & =r_{i+\frac{1}{2}}^{d-1}\left(v_{i+\frac{1}{2}}^{+} Y_{i}-v_{i+\frac{1}{2}}^{-} Y_{i+1}\right)-r_{i-\frac{1}{2}}^{d-1}\left(v_{i-\frac{1}{2}}^{+} Y_{i-1}-v_{i-\frac{1}{2}}^{-} Y_{i}\right), i=2, \cdots, M-1 \\
h r_{M}^{d-1}\left((\nabla \cdot)_{r}^{h}(v Y)\right)_{M} & =r_{M+\frac{1}{2}}^{d-1}\left(v_{M+\frac{1}{2}}^{+} Y_{M}-v_{M+\frac{1}{2}}^{-} Y_{M+\frac{1}{2}}\right)-r_{M-\frac{1}{2}}^{d-1}\left(v_{M-\frac{1}{2}}^{+} Y_{M-1}-v_{M-\frac{1}{2}}^{-} Y_{M}\right)
\end{aligned}
$$

where $x^{+}=\frac{1}{2}(|x|+x)$ and $x^{-}=\frac{1}{2}(|x|-x)$ and $Y_{M+\frac{1}{2}}$ is given by the boundary condition at $r=R$.

\subsection{Parametrization}

Values of most of all parameters used in the model were not directly given in the literature. By crosschecking data available in the literature, we could solely deduce "realistic" values of the model parameters. Consequently, basing ourselves on biological observations, we defined a set of rules to prescribe the model dynamics. We determined parameter values "by hand" so that the simulation results satisfy all these rules.

The basis of rules is a set of biological facts that enabled to limit the range of the "realistic" values of the model parameters. If this method did not make it possible to determine single set of parameter values, it enabled us to define sets of parameter values that led to realistic model dynamics. These biological rules are given below.

(1) A tumor can grow until a diameter of 2 or $3 \mathrm{~mm}$ without developing angiogenesis [23, 25].

(2) In absence of new vascularization, the tumor stays dormant [25].

(3) On the small part of healthy tissue considered here, the mean oxygen concentration is assumed to be constant in time and space.

(4) In the hot spots of a tumor, the part of proliferant cells between tumor cells is of 20 to $30 \%$ [47,54].

(5) Resistance mechanisms make the tumor start growing again after 6 to 8 month in the MTD protocol [27].

(6) Metronomic chemotherapies seem to be more efficient than MTD chemotherapies after 8 to 9 months [18]. 
A "reference" set of parameter values. Distances are given in 1/10 millimeters, time in weeks.

\begin{tabular}{|c|c|c|}
\hline Parameters & Reference value & Meaning \\
\hline$D_{\mathrm{O}}$ & 0.1 & Diffusion coefficient of oxygen in the tissues \\
\hline$\delta_{\mathrm{O}}$ & 0.005 & Coefficient of elimination of the oxygen \\
\hline $\mathrm{O}_{2}^{\max }$ & 1 & Normal concentration of $\mathrm{O}_{2}$ in the tissue \\
\hline$D_{\mathrm{V}}$ & 2 & Diffusion coefficient for $\mathrm{A}_{n g}$ \\
\hline$\delta_{\mathrm{V}}$ & $210^{-4}$ & Coefficient of spontaneous degradation \\
\hline$\alpha_{\mathrm{V}}$ & $\begin{array}{c}410^{-3} \\
0\end{array}$ & $\begin{array}{l}\text { Coefficient of } \mathrm{A}_{n g} \text { production by quiescent cells (vascular growth) } \\
\text { Coefficient of } \mathrm{A}_{n g} \text { production by quiescent cells (avascular growth) }\end{array}$ \\
\hline$\alpha_{\mathrm{EC}}$ & 40 & Proliferation coefficient: $\mathrm{EC}_{n a} \rightarrow \mathrm{EC}_{n a}$ \\
\hline$s_{\mathrm{A}_{n g}, \mathrm{EC}}$ & 0.2 & Threshold on $\mathrm{A}_{n g}$ that control EC proliferation \\
\hline$r_{\mathrm{A}_{n g}, \mathrm{EC}}$ & $10^{3}$ & Transfer parameter involved in the $\mathrm{A}_{n g}$ control of EC proliferation \\
\hline$\mu_{\text {inst }}$ & 0.4 & Transfer coefficient: $\mathrm{EC}_{a} \rightarrow \mathrm{EC}_{n a}$ \\
\hline$\mu_{\text {stab }}$ & 20 & Transfer coefficient: $\mathrm{EC}_{n a} \rightarrow \mathrm{EC}_{a}$ \\
\hline $\mathrm{EC}_{\min }$ & 0.5 & Threshold involved in the transfer $\mathrm{EC}_{n a} \rightarrow \mathrm{EC}_{a}$ \\
\hline$\delta_{\mathrm{EC}}$ & 0.04 & Coefficient of $\mathrm{EC}_{n a}$ degradation \\
\hline$\beta_{\mathrm{P}, \mathrm{EC}_{n a}}$ & 4 & Coefficient of $\mathrm{EC}_{n a}$ degradation by proliferating cells $\mathrm{P}$ \\
\hline$\beta_{\mathrm{Q}, \mathrm{EC}_{n a}}$ & 8 & Coefficient of $\mathrm{EC}_{n a}$ degradation by quiescent cells $\mathrm{Q}$ \\
\hline$\beta_{\mathrm{D}, \mathrm{EC}_{n a}}$ & 40 & Coefficient of $\mathrm{EC}_{n a}$ degradation by dead cells $\mathrm{D}$ \\
\hline$D_{\mathrm{EC}}$ & 1 & Diffusion coefficient for $\mathrm{EC}_{a}$ \\
\hline$\alpha_{\text {recol }}$ & 10 & Recolonization rate of $\mathrm{EC}_{a}$ \\
\hline$\beta_{\mathrm{P}, \mathrm{EC} a}$ & 13 & Coefficient of $\mathrm{EC}_{a}$ degradation by proliferating cells $\mathrm{P}$ \\
\hline$\beta_{\mathrm{Q}, \mathrm{EC}_{a}}$ & 36 & Coefficient of $\mathrm{EC}_{a}$ degradation by quiescent cells $\mathrm{Q}$ \\
\hline$\beta_{\mathrm{D}, \mathrm{EC}_{a}}$ & 140 & Coefficient of $\mathrm{EC}_{a}$ degradation by dead cells $\mathrm{D}$ \\
\hline$\alpha_{\mathrm{P}}$ & 0.5 & Proliferation rate of $\mathrm{P}$ \\
\hline$\gamma_{\mathrm{PQ}}$ & 3 & Transfer coefficient $\mathrm{P} \rightarrow \mathrm{Q}$ acting when $\mathrm{O}_{2}$ small \\
\hline$\gamma_{\mathrm{QP}}$ & 3 & Transfer coefficient $\mathrm{Q} \rightarrow \mathrm{P}$ acting when $\mathrm{O}_{2}$ large \\
\hline$\gamma_{\mathrm{QD}}$ & 3 & Transfer coefficient $\mathrm{Q} \rightarrow \mathrm{D}$ acting when $\mathrm{O}_{2}$ small \\
\hline$\gamma_{\mathrm{MD}}$ & 3 & Transfer coefficient $\mathrm{M} \rightarrow \mathrm{D}$ acting when $\mathrm{O}_{2}$ small \\
\hline$h_{\mathrm{PQ}}$ & 0.38 & $\mathrm{O}_{2}$ threshold involved in the transfer $\mathrm{P} \rightarrow \mathrm{Q}$ \\
\hline$h_{\mathrm{QP}}$ & 0.45 & $\mathrm{O}_{2}$ threshold involved in the transfer $\mathrm{Q} \rightarrow \mathrm{P}$ \\
\hline$h_{\mathrm{QD}}$ & 0.18 & $\mathrm{O}_{2}$ threshold involved in the transfer $\mathrm{Q} \rightarrow \mathrm{D}$ \\
\hline$h_{\mathrm{MD}}$ & 0.25 & $\mathrm{O}_{2}$ threshold involved in the transfer $\mathrm{M} \rightarrow \mathrm{D}$ \\
\hline$\delta_{\text {infl }}$ & 8.333 & Elimination coefficient of dead cells by inflammatory system \\
\hline$\eta_{P}$ & $10^{-3}$ & Efficiency coefficient of the drug on P \\
\hline$\eta_{\mathrm{EC}}$ & 0.14 & Efficiency coefficient of the drug on $\mathrm{EC}_{n a}$ \\
\hline$\alpha_{r}$ & $1.110^{-4}$ & Drug resistance coefficient of $\mathrm{P}$ \\
\hline$r_{\text {tumor }}$ & 1 & Initial size of the tumor \\
\hline $\mathrm{C}_{\min , \mathrm{CT}}$ & 950 & Efficiency threshold on the cytotoxic effect of the drug \\
\hline $\mathrm{C}_{\mathrm{min}, \mathrm{AA}}$ & 655 & Efficiency threshold on the anti-angiogenic effect of the drug \\
\hline
\end{tabular}

\section{Results}

\subsection{Without treatment}

First, simulations without treatment were made with this model of tumor growth. We have started by reproducing and comparing the growth of tumors able to produce angiogenic factors and thus new vascularization, we will refer as vascular tumor growth, and tumors not able to produce angiogenic factors, we will refer as avascular 
tumor growth. Of course, a tumor able to produce angiogenic factors starts its growth without modifying the vascularization thus it starts with an avascular growth.

We recall that Figure 3 presents the initial tumor for all the following simulations. Particularly, we assume that the tumor has an initial diameter of 0.2 millimeter and is centered in the domain (so centered at $r=0$ ). Figure 4 presents the time evolution of the tumor in an avascular setting $\left(\alpha_{V}=0\right)$ and in a vascular setting $\left(\alpha_{V}>0\right)$. Times are in weeks and distances in millimeters. We observe that in both cases, proliferant cells reproduce and make the tumor grow until the vascularization is not sufficient to provide energy for every cell. Part of the proliferant tumor cells becomes quiescent (from $t \geq 4$ ) and then die (from $t \geq 7$ ). Then in the avascular case, the elimination of dead cells by the immune system compensates with the proliferation of proliferant cells and the tumor stops growing (from $t \geq 15$ ) and the whole system converges to a stationary state.

Meanwhile in the vascular case, once quiescent cells appeared, they produce angiogenic factors which triggers proliferation of endothelial cells. The endothelial cells thus settle at the periphery of the tumor, supplying oxygen to the tumor and allowing growth to pursue.

We observe that the tumor grows until it reaches a maximum diameter between 2 and $3 \mathrm{~mm}$. The tumor does not shrink, it stays still waiting for better conditions for growing. This result is consistent with the biological literature $[32,41,59]$.

Figure 5 compares the dynamics for avascular and vascular tumors. We can observe that rules (1)-(4) have been here respected.

\subsection{Comparison of MTD and metronomic chemotherapies}

Let us now test what is the effect of chemotherapy on the tumor growth. The treatment starts at the beginning of the 15th week. We compare two different types of protocol classically used for unresectable glioblastoma. The Maximal Tolerated Dose (MTD) protocol is the protocol described in [46]. The drug (Temozolomide) is administered with 5 intravenous injections of $200 \mathrm{mg} / \mathrm{m}^{2} /$ day at the first 5 days of a cycle. The cycle is assumed to last for 21 days. In the sequel, the standard patient is supposed to have a body area around $1.7 \mathrm{~m}^{2}$. We simulate here the case where 12 cycles of chemotherapy are administered.

The metronomic protocol is taken from [4]. The drug (Temozolomide) is per os (i.e. orally) administered each day during 42 days at a dose of $65 \mathrm{mg} / \mathrm{m}^{2} /$ day or $85 \mathrm{mg} / \mathrm{m}^{2} /$ day, followed by a pause of 7 days. The number of cycles of chemotherapy is not limited in that case.

Figure 6 compares the evolution of the tumor volume depending on the type of protocol used. We can notice that the MTD protocol is clearly more efficient than the metronomic one at the beginning of the treatment, but due to resistance mechanisms, the cytotoxic effect of the MTD chemotherapy decreases after few cycles of treatment. Thus the metronomic protocol becomes more efficient than the MTD one after approximately 8 months. The tumor volume decreases in the metronomic protocol is mainly due to anti-angiogenic effect and thus is less sensible to acquired resistances. However, the decrease of the tumor volume in the metronomic protocol is very slow. We also note that the metronomic treatment fails at doses too low. It is interesting to observe that at the dose $65 \mathrm{mg} / \mathrm{m}^{2}$ /day the tumor volume still grows though very slowly. This is a consequence of the probable existence of a biological threshold below which the drug looses its efficiency. In the model, this fact is expressed through the presence of thresholds $\mathrm{C}_{\min , \mathrm{CT}}$ and $\mathrm{C}_{\min , \mathrm{AA}}$. The values of these thresholds are determining to establish which dose is the most adapted to minimize the tumor size at large time scale and here they are just set to verify rules (5) and (6). In [39], Klement et al studied the cytotoxic and antiangiogenic effects of various chemotherapeutic agents proving that the threshold of efficacy is much lower for the anti-angiogenic effect than for the cytotoxic effect. This is translated in our model by $\mathrm{C}_{\min , \mathrm{CT}} \gg \mathrm{C}_{\min , \mathrm{AA}}$. However without further medical knowledge it is impossible to determine from this model what is the best dose of chemotherapeutic agent to administrate. Nevertheless this model confirms the "metronomic hypothesis" that the anti-angiogenic effect of chemotherapeutic agents should be privileged to limit the growth of a tumor. This can be done by reducing the pause between two cycles of chemotherapy which implies necessarily lower doses of chemotherapeutic agent during the cycle. Indeed the fact that the metronomic protocol wins at large scale 


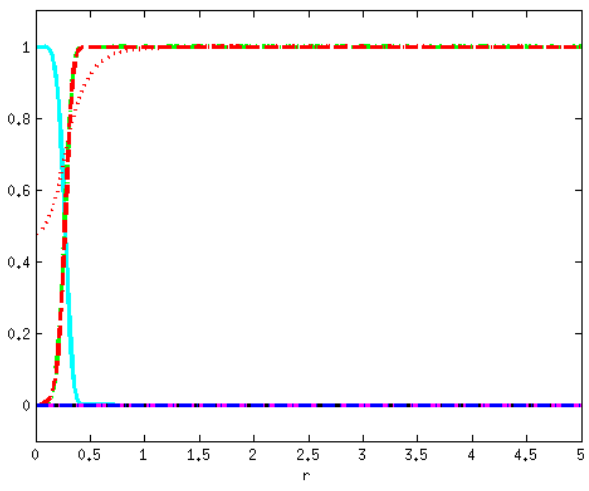

(a) $t=4$ avascular

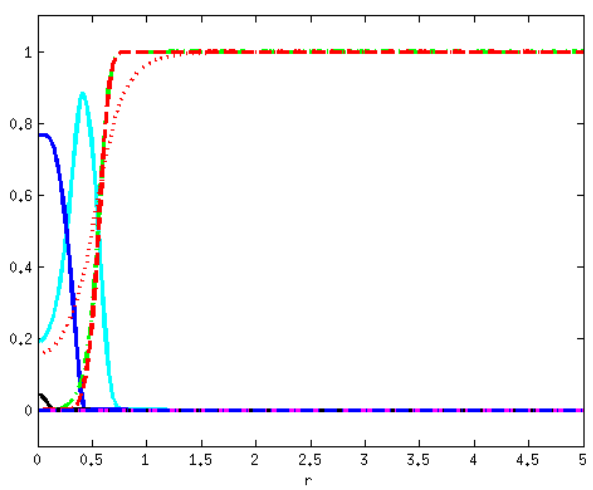

(c) $t=7$ avascular

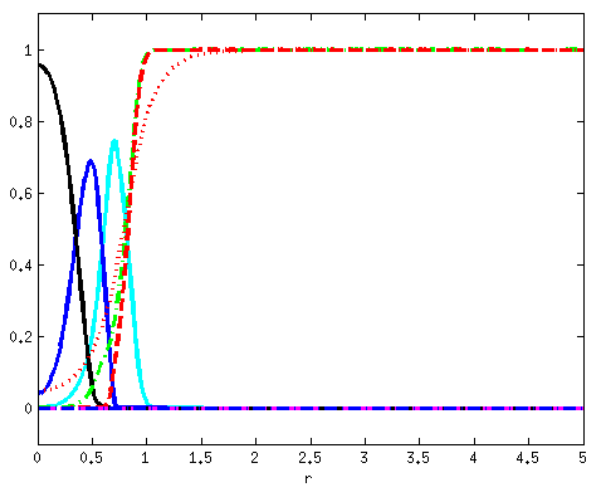

(e) $t=10$ avascular

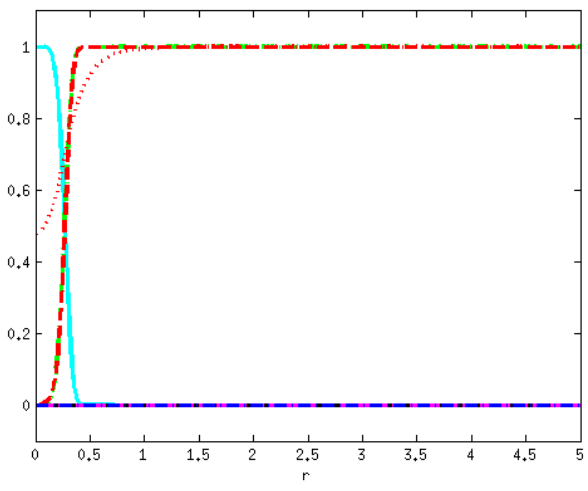

(b) $t=4$ vascular

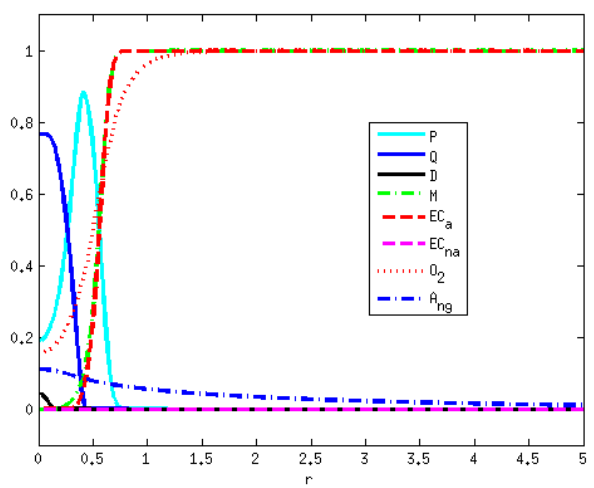

(d) $t=7$ vascular

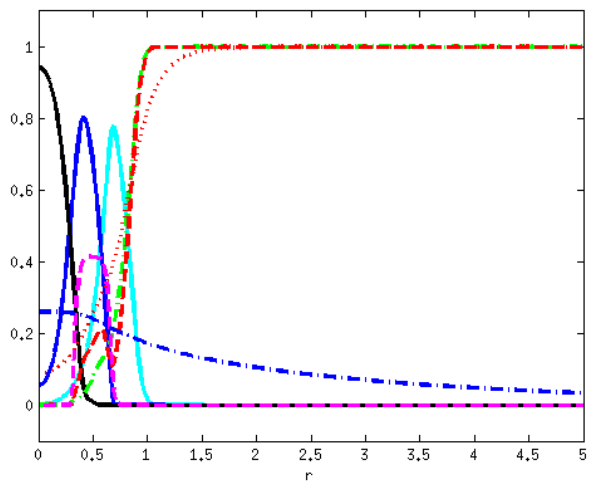

(f) $t=10$ vascular

Figure 4. Comparison of avascular (left) and vascular (right) tumor growth. Times are given in weeks and distances in millimeters. 


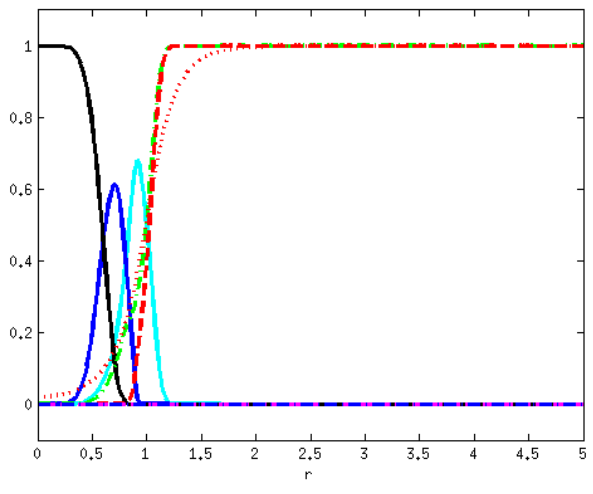

(g) $t=15$ avascular

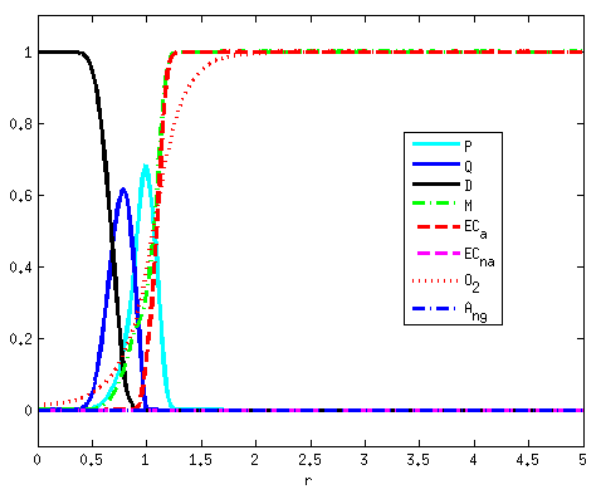

(i) $t=20$ avascular

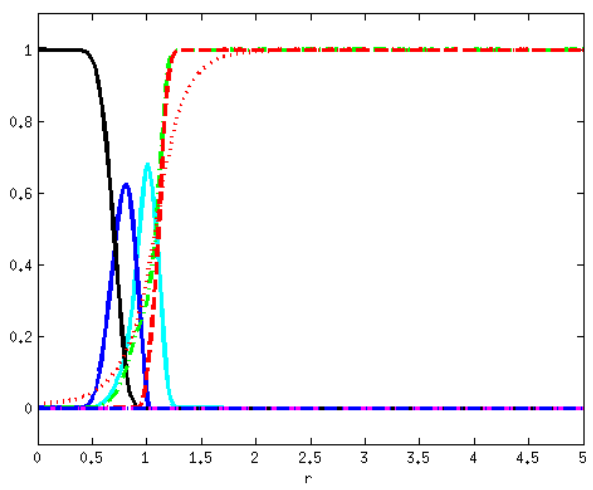

(k) $t=30$ avascular

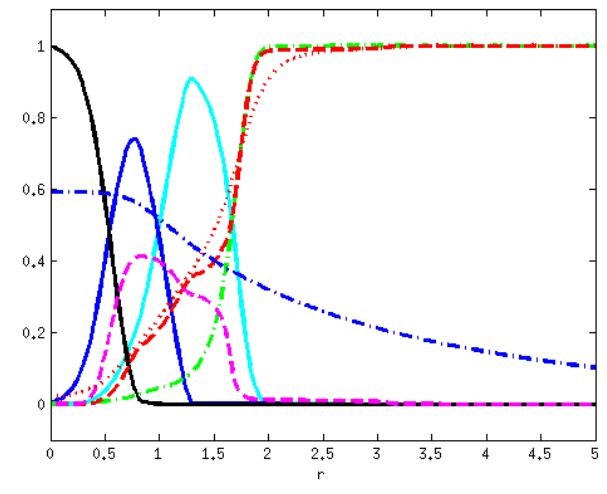

(h) $t=15$ vascular

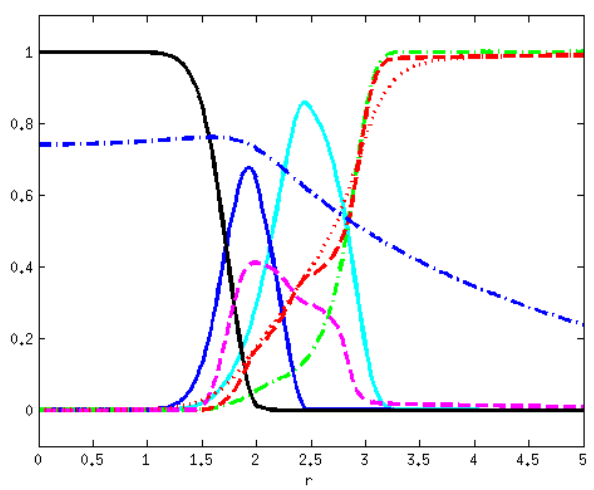

(j) $t=20$ vascular

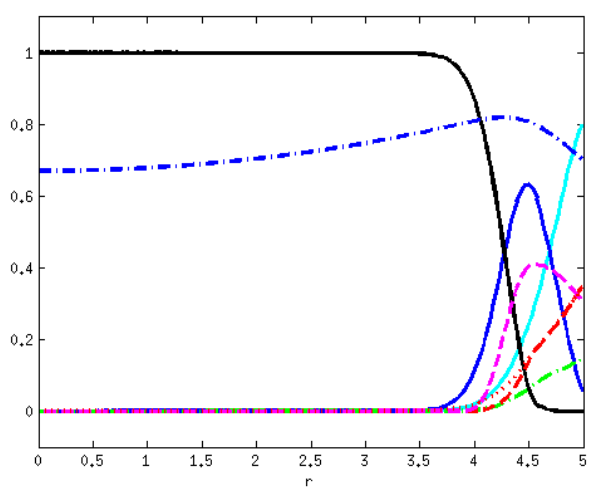

(l) $t=30$ vascular

Figure 4. Comparison of avascular (left) and vascular (right) tumor growth. Times are given in weeks and distances in millimeters. (continued) 


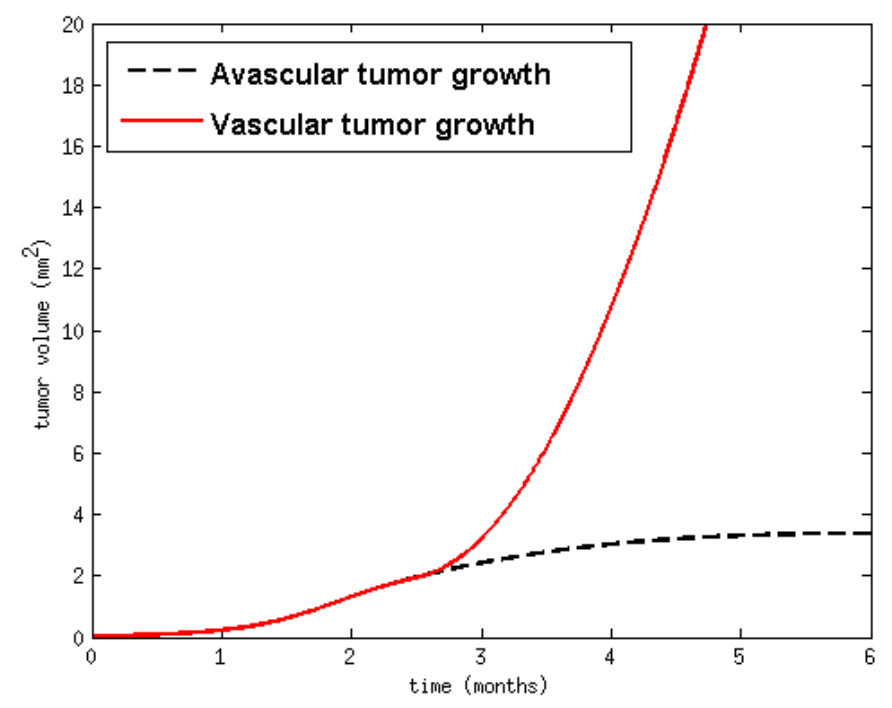

FiguRE 5. Comparison of tumor volumes $(\mathrm{P}+\mathrm{Q}+\mathrm{D})$ depending on time for "vascular" and "avascular" tumor growth. Time is in months and sizes are in $\mathrm{mm}^{2}$. We show here that angiogenesis promotes tumor growth, which would otherwise be stabilized.

relies first on the fact that endothelial cells recovers from treatment much faster than cancer cells and second on the fact that endothelial cells are less susceptible of mutation and thus of resistance to treatment. Therefore the reduction of the tumor size in the metronomic protocol is due to the anti-angiogenic effect of chemotherapy (the tumor is "suffocated") which disappear when pauses between cycles are too long. In the MTD protocol, the anti-angiogenic effect is weak because of long pauses and the cytotoxic effect lower with time due to the appearance of resistances. Adding more MTD chemotherapy cycles is thus useless, the metronomic protocol still works better.

Note that the pharmacodynamics as well as the pharmacokinetics of the drugs have not been taken into account in this work. The concentration $C(t)$ of the drug is simply linked to the administered dose as shown in Figure 6 (Right). It is well known in particular that the PK of intravenous injections and per os administration are very different. The introduction of the PK/PD of the drug will be fundamental in view of clinical applications of the model.

\section{Conclusion And discussion}

Nowadays, the paradigm of metronomic chemotherapies is a challenge in oncology, it would have good efficiency while limiting toxicities. However, to improve the efficiency of a metronomic chemotherapy, it is essential to be able to answer some clinical questions: What is a small dose? What is the most appropriate dose? What is the best rhythm for the drug administration? How chronology of drug administration between metronomic chemotherapy and anti-angiogenic impacts the effect of the therapy? This present work is a first step in the construction of a numerical tool able to give answers to all these questions.

The model proposed here, that combines the dynamics of tumor cells and endothelial cells in interaction thanks to molecules as oxygen and angiogenic factors, enables us to reproduce the expected efficiency of MTD and metronomic schedule in the case of Temozolomide chemotherapy. These first results are hopeful. Of course, this model is not restricted to the administration of Temozolomide and can be adapted to any kind of chemotherapy, in particular to any cocktail of anti-cancer agents. To take into account the clinical reality of 

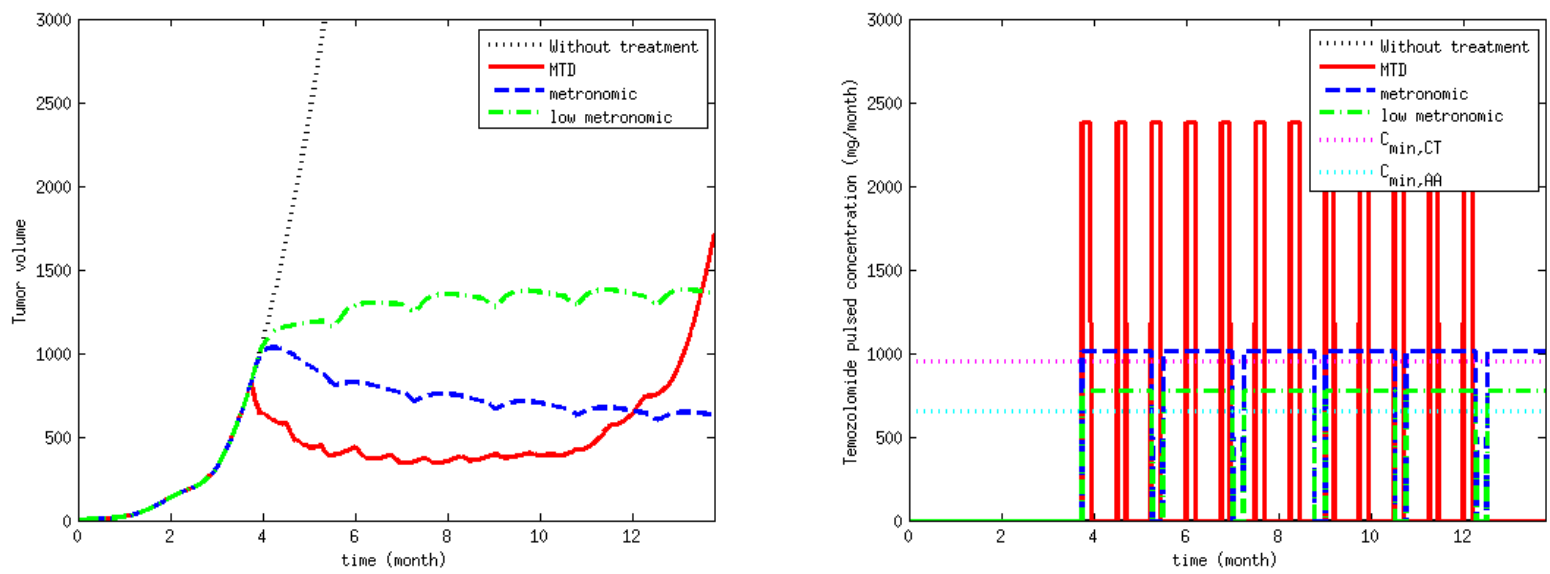

Figure 6. Left . Comparison of tumor volumes $(\mathrm{P}+\mathrm{Q}+\mathrm{D})$ without treatment, with MTD chemotherapy and with metronomic chemotherapy depending on the time in month. Volumes are given in $\mathrm{mm}^{2}$. Right. The pulsed concentration $t \mapsto C(t)$ of Temozolomide for the different protocols. Let us remark that the total doses mentioned in the various protocols can be recognized when integrating these pulse concentrations over a day (taking into account that the time unit is the week).

the drugs' effects, we will have to enrich the model using a pharmacokinetics and pharmacodynamics (PK/PD) model for each drug administrated.

Furthermore, we exhibit here a good set of parameter values suitable for a glioblastoma non treated or treated by Temozolomide. In practice, there exists a huge variability of these parameters due to both the type of cancer or the diversity of metabolism in the patients. Thus a clinical transfer of such a huge system requires to reduce the set of parameters. A careful parametric study of the system as well as new biological quantitative rules will help us to identify a small set of parameters crucial for such dynamics.

Note that we present here results for tumors that are radially distributed, but of course the model could handle any kind of tumor shape, just by changing initial condition. In the same way, it could be interesting to take into account the heterogeneity of the tumor, by considering anisotropic diffusion of the different quantities.

\section{REFERENCES}

[1] N. André, L. Padovani, and E. Pasquier. Metronomic scheduling of anticancer treatment: the next generation of multitarget therapy? Future Oncol., 3:385-94, 2011.

[2] D. Balding and D. McElwain. A mathematical model of tumour-induced capillary growth. J Theor Biol, 114(1):53-73, May 1985.

[3] D. Barbolosi and A. Iliadis. Optimizing drug regimens in cancer chemotherapy: a simulation study using a PK-PD model. Comput Biol Med, 31:157-172, 2001.

[4] S. Baruchel, M. Diezi, D. Hargrave, D. Stempak, J. Gammon, A. Moghrabi, M. Coppes, C. Fernandez, and E. Bouffet. Safety and pharmacokinetics of temozolomide using a dose-escalation, metronomic schedule in recurrent paediatric brain tumours. Eur. J. Cancer, 42:2335-2342, 2006.

[5] S. Benzekry, N. André, A. Benabdallah, J. Ciccolini, C. Faivre, F. Hubert, and D. Barbolosi. Modelling the impact of anticancer agents on metastatic spreading. Mathematical Modelling of Natural Phenomena, 7(1):306-336, 01/2012 2012.

[6] S. Benzekry, G. Chapuisat, J. Ciccolini, A. Erlinger, and F. Hubert. A new mathematical model for optimizing the combination between antiangiogenic and cytotoxic drugs in oncology. Comptes Rendus de l'Académie des Sciences - Series I - Mathematics, 350:23-28, 2012 . 
[7] F. Billy, J. Clairambault, O. Fercoq, S. Gaubert, T. Lepoutre, T. Ouillon, and S. Saito. Synchronisation and control of proliferation in cycling cell population models with age structure. Math. Comp. Simul., 2012. in press, available on line Apr. 2012.

[8] F. Billy, B. Ribba, O. Saut, H. Morre-Trouilhet, T. Colin, D. Bresch, J.-P. Boissel, E. Grenier, and J.-P. Flandrois. A pharmacologically based multiscale mathematical model of angiogenesis and its use in investigating the efficacy of a new cancer treatment strategy. Journal of Theoretical Biology, 260(4):545-562, 2009.

[9] D. Bresch, T. Colin, E. Grenier, B. Ribba, and O. Saut. Computational modeling of solid tumor growth: the avascular stage. SIAM J. Sci. Comput., 32(4):2321-2344, 2010.

[10] C. Breward, H. Byrne, and C. Lewis. A multiphase model describing vascular tumour growth. Bull Math Biol, 65(4):609-640, Jul 2003.

[11] T. Browder, C. Butterfield, B. Kraling, B. Shi, B. Marshall, M. O'Reilly, and J. Folkman. Antiangiogenic scheduling of chemotherapy improves efficacy against experimental drug-resistant cancer. Cancer Res., 60(7):1878-1886, 2000.

[12] H. Byrne and M. Chaplain. Growth of nonnecrotic tumors in the presence and absence of inhibitors. Math Biosci, 130(2):151181, Dec 1995.

[13] H. Byrne and M. Chaplain. Mathematical models for tumour angiogenesis: numerical simulations and nonlinear wave solutions. Bull Math Biol, 57(3):461-486, May 1995.

[14] M. Chaplain. The mathematical modelling of tumour angiogenesis and invasion. Acta Biotheor, 43(4):387-402, Dec 1995.

[15] M. Chaplain, S. Giles, B. Sleeman, and R. Jarvis. A mathematical analysis of a model for tumour angiogenesis. J Math Biol, 33(7):744-770, 1995.

[16] M. Chaplain, S. McDougall, and A. Anderson. Mathematical modeling of tumor-induced angiogenesis. Annu Rev Biomed Eng, Apr 2006.

[17] M. Chaplain and A. Stuart. A model mechanism for the chemotactic response of endothelial cells to tumour angiogenesis factor. IMA J Math Appl Med Biol, 10(3):149-168, 1993.

[18] C. Chen, T. Xu, Y. Lu, J. Chen ans S. Wu. The efficacy of temozolomide for recurrent glioblastoma multiforme. Eur J Neurol., 20(2):223-30, 2013.

[19] E. De Angelis and L. Preziosi. Advection-diffusion models for solid tumour evolution in vivo and related free boundary problem. Math. Models Methods Appl. Sci., 10(3):379-407, 2000.

[20] A. Ergun, K. Camphausen, and L. M. Wein. Optimal scheduling of radiotherapy and angiogenic inhibitors. Bull Math Biol, 65(3):407-424, 2003.

[21] R. Eymard, T. Gallouët, and R. Herbin. Finite volume methods. In Handbook of numerical analysis, Vol. VII, Handb. Numer. Anal., VII, pages 713-1020. North-Holland, Amsterdam, 2000.

[22] C. Faivre, D. Barbolosi, and A. Iliadis. On determining the mtd for phase I trials in pediatric oncology. Curr Top Med Chem, Sep 2012.

[23] J. Folkman. Tumor angiogenesis: therapeutic implications. N Engl J Med, 285(21):1182-1186, Nov 1971.

[24] J. Folkman. Anti-angiogenesis : New concept for therapy of solid tumors. Annals of Surgery, 175(3), 1971-1972.

[25] J. Folkman. What is the evidence that tumors are angiogenesis dependent? Journal of the National Cancer Institute, 82(1), 1990.

[26] H. B. Frieboes, M. E. Edgerton, J. P. Fruehauf, F. R. A. J. Rose, L. K. Worrall, R. A. Gatenby, M. Ferrari, and V. Cristini. Prediction of drug response in breast cancer using integrative experimental/computational modeling. Cancer Res, 69(10):44844492, May 2009.

[27] M.R. Gilbert, M. Wang, K.D. Aldape, R. Stupp, M.E. Hegi, K.A. Jaeckle, T.S. Armstrong, J.S. Wefel, M. Won, D.T. Blumenthal, A. Mahajan, C.J. Schultz, S. Erridge, B. Baumert, K.I. Hopkins, T. Tzuk-Shina, P.D. Brown, A. Chakravarti, W.J. Curran, M.P. Mehta. Dose-Dense Temozolomide for Newly Diagnosed Glioblastoma: A Randomized Phase III Clinical Trial. J Clin Oncol. 2013.

[28] G.W. Swan. Role of optimal control theory in cancer chemotherapy. Mathematical Biosciences, 101:237-284, 1990.

[29] P. Hahnfeldt, J. Folkman, and L. Hlatky. Minimizing long-term tumor burden: the logic for metronomic chemotherapeutic dosing and its antiangiogenic basis. J Theor Biol, 220(4):545-554, Feb 2003.

[30] P. Hahnfeldt, D. Panigrahy, J. Folkman, and L. Hlatky. Tumor development under angiogenic signaling: a dynamical theory of tumor growth, treatment response, and postvascular dormancy. Cancer Res, 59(19):4770-4775, Oct 1999.

[31] D. Hanahan, G. Bergers, and E. Bergsland. Less is more, regularly: metronomic dosing of cytotoxic drugs can target tumor angiogenesis in mice. J. Clin. Invest., 105:1045-1047, 2000.

[32] D. Hanahan and J. Folkmann. Patterns and emerging mechanisms of the angiogenic switch during tumorigenesis. Cell, 86:35364, 1996.

[33] C. Hogea, B. Murray, and J. Sethian. Simulating complex tumor dynamics from avascular to vascular growth using a general level-set method. J Math Biol, 53(1):86-134, Jul 2006.

[34] A. Iliadis and D. Barbolosi. Optimizing drug regimens in cancer chemotherapy by an efficacy-toxicity mathematical model. Comput Biomed Res, 33:211-226, 2000.

[35] T. L. Jackson. Vascular tumor growth and treatment: consequences of polyclonality, competition and dynamic vascular support. J. Math. Biol., 44(3):201-226, 2002. 
[36] T. L. Jackson and H. M. Byrne. A mathematical model to study the effects of drug resistance and vasculature on the response of solid tumors to chemotherapy. Math. Biosci., 164(1):17-38, 2000.

[37] R. S. Kerbel. Inhibition of tumor angiogenesis as a strategy to circumvent acquired resistance to anti-cancer therapeutic agents. Bioessays, 13:31-36, 1991.

[38] P. Kevrekidis, N. Whitaker, D. Good, and G. Herring. Minimal model for tumor angiogenesis. Phys Rev E Stat Nonlin Soft Matter Phys, 73(6 Pt 1):061926, Jun 2006.

[39] G. Klement, S. Baruchel, J. Rak, S. Man, K. Clark, D. Hicklin, P. Bohlen, and R. Kerbel. Continuous low-dose therapy with vinblastine and vegf receptor-2 antibody induces sustained tumor regression without overt toxicity. J Clin Invest., 105(8):R15R24, 2000.

[40] U. Ledzewicz, H. Maurer, and H. Schättler. Optimal and suboptimal protocols for a mathematical model for tumor antiangiogenesis in combination with chemotherapy. Mathematical Biosciences and Engineering, 8:307-323, 2011.

[41] M. Tubiana Klaas breur medal lecture 1985. the growth and progression of human tumors: implications for management strategy. Radiother. Oncol., 6(3):167-84, 1986.

[42] D. Majumder. Tumor angiogenesis based analytical model for the assessment of MCT and MTD chemotherapeutic strategies in cancer. J. Biol. Systems, 18(4):749-761, 2010.

[43] D. Majumder and A. Mukherjee. Patho-physiologically based logistics for treatment of cancer. Journal of Biological Systems, 14(4):631-650, 2006.

[44] C. Meille, J. Gentet, D. Barbolosi, N. André, F. Doz, and A. Iliadis. New adaptive method for phase i trials in oncology. Clinical Pharmacology and Therapeutics, 83:873-881, 2008.

[45] A. Mukherjee and D. Majumder. Mathematical modelling for the assessment of the effect of drug application delays in metronomic chemotherapy of cancer due to physiological constraints. Biosystems, 91(1):108-116, Jan 2008.

[46] H. Nicholson, C. Kretschmar, M. Krailo, M. Bernstein, R. Kadota, D. Fort, H. Friedman, M. Harris, N. Tedeschi-Blok, C. Mazewski, J. Sato, and G. Reaman. Phase 2 study of temozolomide in children and adolescents with recurrent central nervous system tumors: a report from the children's oncology group. Cancer, 110(7):1542-50, 2007.

[47] R. Nishimura, T. Osako, Y. Okumura, M. Hayashi, Y. Toyozumi, N. Arima Ki-67 as a prognostic marker according to breast cancer subtype and a predictor of recurrence time in primary breast cancer, Exp Ther Med., 1(5):747-754, 2010.

[48] A. d. Onofrio. Rapidly acting antitumoral antiangiogenic therapies. Phys Rev E Stat Nonlin Soft Matter Phys, 76(3 Pt 1):031920, 2007.

[49] A. d. Onofrio and A. Gandolfi. Tumour eradication by antiangiogenic therapy: analysis and extensions of the model by Hahnfeldt et al. (1999). Math. Biosci., 191:159-184, 2004.

[50] A. d. Onofrio and A. Gandolfi. A family of models of angiogenesis and anti-angiogenesis anti-cancer therapy. Math Med Biol, 26(1):63-95, 2009.

[51] M. Orme and M. Chaplain. A mathematical model of vascular tumour growth and invasion. Math. Comput. Modelling, 23(10):43-60, 1996.

[52] E. Pasquier, M. Kavallaris, and N. André. Metronomic chemotherapy: new rationale for new directions. Nat Rev Clin Oncol, 7(8):455-465, Aug 2010.

[53] M. Plank, B. Sleeman, and P. Jones. A mathematical model of tumour angiogenesis, regulated by vascular endothelial growth factor and the angiopoietins. J Theor Biol, 229(4):435-454, Aug 2004.

[54] Q. Romero, P.O. Bendahl, M. Klintman, N. Loman, C. Ingvar, L. Rydén, C. Rose, D. Grabau, S. Borgquist. Ki67 proliferation in core biopsies versus surgical samples - a model for neo-adjuvant breast cancer studies. BMC Cancer.,7; 11:341, 2011.

[55] I. Stamper, H. Byrne, M. Owen, and P. Maini. Modelling the role of angiogenesis and vasculogenesis in solid tumour growth. Bull Math Biol, 69(8):2737-2772, Nov 2007.

[56] A. Swierniak, A. Polanski, and M. Kimmel. Optimal control problems arising in cell-cycle-specific cancer chemotherapy. Cell Prolif, 29(3):117-139, Mar 1996.

[57] Y. Tao, N. Yoshida, and Q. Guo. Nonlinear analysis of a model of vascular tumour growth and treatment. Nonlinearity, 17:867-895, 2004.

[58] B. You, C. Meille, D. Barbolosi, B. Tranchand, J. Guitton, C. Rioufol, A. Iliadis, and G. Freyer. A mechanistic model predicting hematopoiesis and tumor growth to optimize docetaxel + epirubicin (et) administration in metastatic breast cancer (mbc): Phase i trial. In ASCO Annual Meeting, Journal of Clinical Oncology, volume 25, Chicago, 2007.

[59] B. Zetter. Angiogenesis and tumor metastasis. 1nnu. Rev. Med., 49:407-84, 1998.

[60] X. Zheng, S. Wise, and V. Cristini. Nonlinear simulation of tumor necrosis, neo-vascularization and tissue invasion via an adaptive finite-element/level-set method. Bull Math Biol, 67(2):211-259, Mar 2005. 